\title{
Toroidal Geometry and Plasma Column Displacement Corrections in the Analysis of Mirnov Oscillations
}

\author{
M.S.T. Araújo, A. Vannucci, and K.A. Oliveira \\ Physics Institute, University of São Paulo \\ P.O. Box 66.318, São Paulo, 05315-970, S.P., Brazil
}

Received on 26 June, 2001

\begin{abstract}
The influence of the toroidal geometry, and the plasma column displacement, over the usual tokamak poloidal magnetic field configuration was investigated for circular plasmas through a series of simulations. The results obtained clearly showed the existence of a change in phase modulation of the Mirnov signals, which are not usually considered when the magnetic signals are Fourier analyzed in the frame of cylindrical approximation, that is, by neglecting any possible toroidal effect. Furthermore, if the occasional displacements of the plasma column in tokamaks are considered, some differences on the mode composition are also observed. These discrepancies, however, do not seem to affect the analysis of the magnetic signals as strongly as in the former case, when toroidal effects are neglected.
\end{abstract}

\section{Introduction}

For decades the experimental magnetic signals measured by Mirnov coils have been intensively investigated with the objective of observing the phase modulation and changes in amplitude of the perturbed poloidal magnetic field, during normal tokamak discharges [16]. Almost as a common practice, the pick-up coils are chosen to be installed internally to the tokamak vacuum chamber, equally distributed along the poloidal direction, in a given toroidal cross-section. Thus, when the experimental magnetic signals are Fourier analyzed the influence of the toroidal geometry on these signals are simply ignored. This practice, most of the time, leads to conclusions that are not very accurate, as for example, when one tries to identify the contribution of different precursor MHD modes to the triggering mechanism of plasma instabilities [7-10].

One possible alternative to overcome this problem, which has been proposed elsewhere and that might be used in already existing pick-up coils systems, is to collect the experimental signals from the Mirnov coils, equally spaced along the poloidal direction, and to introduce, thereafter, the necessary toroidal effect corrections [11].

A better approach, however, which appears much more appropriate to us, because it considerably facilitates the analysis of the Mirnov oscillations, is to rebuild the entire magnetic pick-up coils system but now with different angular spacing between neighbor- ing magnetic coils, in such a way the influence of the toroidal geometry on the measured magnetic signals would already be naturally included.

In this article we describe a series of simulations which was carried out in order to demonstrate that, if the Mirnov coils are distributed along a circular cross section of a tokamak, in a convenient way, the measured experimental magnetic signals would already incorporate the toroidal geometry effects, making the correct Fourier analysis of these signals a straightforward task.

In general, the plasma current within a rational surface $\mathrm{r}_{m, n}$, which is usually associated to the development of resistive MHD modes, can be expressed in the form $[11,12]$ :

$$
\tilde{j}=j_{m, n} \cos (\tilde{\xi}) \delta\left(r-r_{m, n}\right)
$$

where $\tilde{\xi}$ is defined by $[11,13]$ :

$$
\tilde{\xi}=m \theta^{*}+n \phi+\delta_{0}-\omega t
$$

and the "Merezhkin" phase correction, due to the toroidal effect, is given by $[11,13]$ :

$$
\theta^{*}=\theta-\lambda \sin \theta
$$

where:

$$
\lambda=\lambda\left(r_{m, n}\right)=\frac{r_{m, n}}{R_{m, n}}\left[\beta_{p}\left(r_{m, n}\right)+\frac{1}{2} l_{i}\left(r_{m, n}\right)+1\right]
$$


In equation (4), $\beta_{p}$ is the ratio between the kinetic and the magnetic poloidal pressures, $l_{i}$ is the plasma internal inductance and $\mathrm{R}_{m, n}$ is the major radius associated to the resonant magnetic surface at $q=m / n$.

In relation to the above equations, if the cylindrical geometry is assumed then the parameter $\lambda=0$, and the geometrical phase asymmetry of the magnetic oscillations disappears. However, by gradually introducing corrections related to the toroidal geometry of the system, the center of the resistive modes (that is, the center of the resonant magnetic surfaces) will not coincide anymore with the geometric center of the vacuum camera due to the Shafranov shift. Therefore, the magnetic flux surfaces as a whole are moved towards to the outside (region of lower toroidal field) and the disturbance currents within the rational magnetic surfaces generate more intense magnetic field within this region. For this reason, the pick up coils that monitor the inner area (region of higher $\mathrm{B}_{\phi}$ ) must have a higher sensitivity than the outer ones, located in the region of lower $\mathrm{B} \phi$.

In this work, the effect of the toroidal geometry on the Mirnov oscillations was studied by considering a simplified model, in which the "Merezhkin" correction $\left(\theta \rightarrow \theta^{*}\right)$ is written in terms of the toroidicity parameter $\lambda$ expressed only as a function of the inverse of the tokamak aspect ratio (calculated at the position of interest), that is:

$$
\lambda(r)=\varepsilon(r)=\frac{r}{R_{0}}
$$

Finally, another effect (of second order) was also investigated, which is related to the change in amplitude and phase modulation of the magnetic signals due to plasma column displacements that are commonly observed during a normal tokamak plasma discharge.

\section{Simulations}

The quantitative evaluation of how the toroidal geometry influences the Fourier analysis of the Mirnov oscillations was carried out by considering the tokamak toroidicity parameter $\left(\varepsilon=\mathrm{r} / \mathrm{R}_{0}\right)$ varying from zero to 0.4 . While for $\varepsilon=0$ the toroidal effect is totally neglected, for values larger than that the toroidal effect can be gradually introduced. The method employed in this evaluation consisted, initially, in simulating a magnetic field signal formed only by the $\mathrm{m}=8 \mathrm{com}-$ ponent. The reason for this choice is that we intended to apply the results of these simulations, afterwards, to the newly constructed Mirnov coils system for the small TBR tokamak $\left(\mathrm{R}_{0}=30 \mathrm{~cm}, \mathrm{a}=10 \mathrm{~cm}, \mathrm{~B}_{\phi}=0.4 \mathrm{~T}\right.$,
$\mathrm{I}_{p}=12 \mathrm{kA}$ and $\left.\tau_{D} 10 \mathrm{~ms}\right)$ running circular plasmas, which is composed of 16 magnetic pick-up coils and distributed along the poloidal direction accordingly to equation 3. Therefore, the position related to each coil would automatically correspond to a null point of the simulated profile.

The spatial configuration of the poloidal perturbed magnetic field, as a function of the equilibrium coordinates $\left(\theta^{*}, \phi\right)$, was calculated at one instant of time (for a given value of the parameter $\varepsilon$ ) accordingly to [14]:

$$
B=B_{0} \cos \left(8 \theta^{*}+n \phi\right)
$$

where $\theta^{*}$ was already defined in equation 3 . Therefore, the poloidal configuration of a simulated magnetic field formed exclusively by the $\mathrm{m}=8$ component using $\varepsilon=0.32$, for example (as for the TBR tokamak), is shown in Fig. 1. As it can be readily noticed, when the toroidal geometry effect is introduced a strong poloidal phase asymmetry is observed, related to a more rapidly oscillating poloidal magnetic field within the region of stronger toroidal magnetic field $(\theta=\pi)$ of the tokamak. For comparison, Fig. 2 exhibits the corresponding configuration obtained for the simulated $\mathrm{m}=8$ magnetic field, but now assuming the cylindrical approximation $(\varepsilon=0)$.

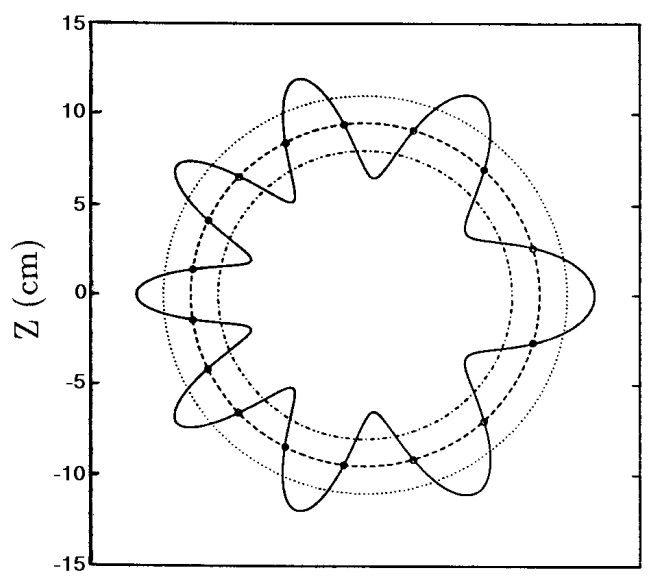

Figure 1. Poloidal configuration of the simulated magnetic field formed only by the $\mathrm{m}=8$ component, considering the toroidal geometry effect. 


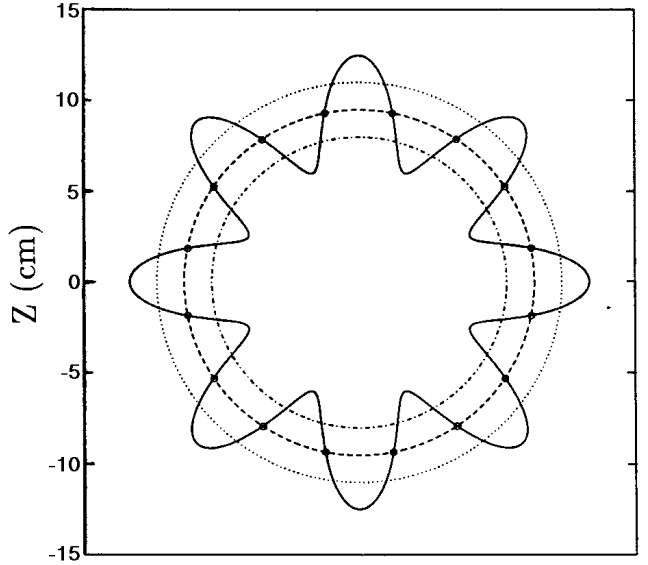

Figure 2. Poloidal configuration of the simulated magnetic field in the cylindrical approximation (neglecting the effects of the toroidal geometry).

Afterwards, we investigate the results obtained when the pure $\mathrm{m}=8$ simulated poloidal magnetic field (associated with the configuration shown in Fig. 1) is Fourier analyzed, by gradually introducing the toroidal geometry effect.

If it is considered that this magnetic configuration is measured with a set of pick up coils positioned along the poloidal direction assuming $\varepsilon=0$ (coils are equally spaced, accordingly to cylindrical approximation), the resulting mode composition as obtained from the Fourier analysis of these signals would yield the diagram shown in Fig. 3a. As it can be clearly observed from this diagram, the $\mathrm{m}=8 \mathrm{MHD}$ mode, in spite of being the only one chosen to form the simulated signal (equation 6), it would be erroneously considered to contribute with only $2.7 \%$ to the total mode spectrum. The dominant components, in this case, would appear to be the $\mathrm{m}=6, \mathrm{~m}=7, \mathrm{~m}=9$ and $\mathrm{m}=10$ modes, more or less symmetrically distributed (in terms of mode intensity) around the $\mathrm{m}=8$.
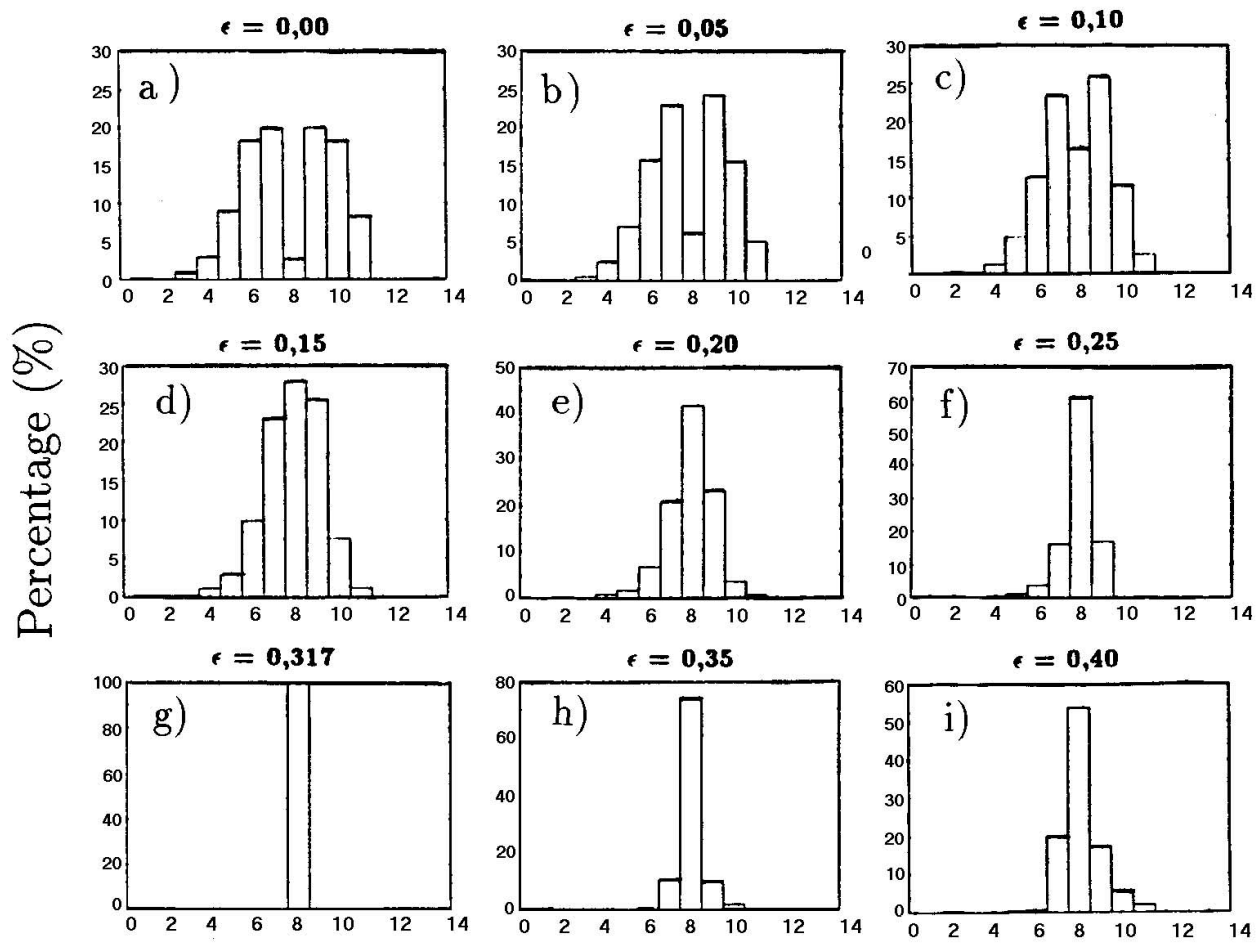

m Modes

Figure 3. Mode composition obtained for: a) $\varepsilon=0.0$, b) $\varepsilon=0.05$, c) $\varepsilon=0.10$, d) $\varepsilon=0.15$, e) $\varepsilon=0.20$, f) $\varepsilon=0.25$, g) $\varepsilon=$ $0.32, \mathrm{~h}) \varepsilon=0.35$ and i) $\varepsilon=0.40$.

If the value $\varepsilon=0.05$ is used (very small toroidal effect is introduced) the decomposition of the modes thereof obtained corresponds to the diagram shown in fig. $3 \mathrm{~b}$. Note that the contribution of the $\mathrm{m}=8 \mathrm{com}-$ ponent is still very small (about $6.1 \%$ of the total mode spectrum) and the dominant modes would be mainly the $\mathrm{m}=7$ and $\mathrm{m}=9$. For $\varepsilon=0.10$ (Fig. 3c) the con- 
tribution of the $\mathrm{m}=8$ component would correspond to $16.3 \%$. This value, however, still represents a secondary contribution of the $\mathrm{m}=8$ to the total mode spectrum, since the $\mathrm{m}=7$ and $\mathrm{m}=9$ components maintain the predominance over all the other modes, each one of them contributing with about $25 \%$ to the total spectrum.

A more significant participation of the $\mathrm{m}=8$ mode to the total mode spectrum starts to become evident for $\varepsilon=0.15$. According to the diagram shown in Fig. $3 \mathrm{~d}$, the $\mathrm{m}=8$ contribution would now correspond to $28 \%$ of the spectrum, followed by smaller contributions of the $\mathrm{m}=7$ and $\mathrm{m}=9$ components. The diagrams shown in Figs. 3e and 3f, on the other hand, correspond to the results obtained for $\varepsilon=0.20$ and $\varepsilon=0.25$, respectively. As it is clearly observed, the predominance of the $\mathrm{m}=8$ component is now strongly accentuated, reaching $41.6 \%$ and $60.8 \%$, respectively, of the total mode spectrum.

Finally, when $\varepsilon=0.32$ is used (which corresponds to the inverse of the aspect ratio of the TBR tokamak, calculated accordingly to the radial positioning of the Mirnov coils), a spectrum composed exclusively by the $\mathrm{m}=8$ mode is then obtained, as shown in Fig $3 \mathrm{~g}$. For larger values of $\varepsilon$, it is verified that the contribution of the $\mathrm{m}=8$ component starts decreasing again, as for $\varepsilon=0.35$ and $\varepsilon=0.4$, reaching $74.3 \%$ and $53.6 \%$ of the total spectrum, respectively (Figs $3 \mathrm{~h}$ and $3 \mathrm{i}$ ).

Now, with the objective of investigating further how significantly the toroidal geometry may affect the Fourier analysis of the Mirnov magnetic signals, another set of simulations was carried out using more realistic poloidal magnetic field configurations. Firstly, the simulated signal was chosen to be composed by a sum of different modes, but mainly of $\mathrm{m}=3$ and $\mathrm{m}=4$ components, accordingly to the diagram shown in Fig. 4. Both line-point curves in Fig. 4a represent the vacuum vessel and the tokamak material limiter, respectively, while the broken-line curve represents the radial positioning of the magnetic coils (which are identified by small circles along the poloidal direction), as indicated by the calculations using $\varepsilon=0.32$. In Fig. $4 \mathrm{~b}$ the corresponding mode-composition, in percentage, can be observed. However, this particular simulated magnetic configuration, as it would be measured by Mirnov system, if the magnetic coils were equally distributed along the poloidal direction ( $\varepsilon=0$ - cylindrical model), is shown in Fig. 5a. Note that the mode decomposition now incorrectly indicates the $\mathrm{m}=3$ as the dominant MHD mode, followed by the $\mathrm{m}=4$ and, less intensively, by the $\mathrm{m}=5, \mathrm{~m}=6$ and $\mathrm{m}=7$ components (Fig. $5 \mathrm{~b}$ ).

Finally, another magnetic field configuration which corresponds to a signal formed by a pure $\mathrm{m}=3$ mode, was also investigated in the frame of our simulations. The spatial structure of the corresponding magnetic signals as measured by the pick up coils, if they were dis- tributed considering the TBR tokamak toroidal geometry, is shown in fig 6a. The Fourier analysis of magnetic signals is shown in Fig. 6b which indicates, as expected, the only presence of the original $\mathrm{m}=3$ component. On the other hand, if the Mirnov coils were considered to be equally distributed along the poloidal direction (neglecting, consequently, any possible toroidal effect) the Fourier decomposition would reveal an unreal mode composition, as shown in Figs $7 \mathrm{a}$ and $7 \mathrm{~b}$. From these figures it can be noted that although the $\mathrm{m}=3 \mathrm{com}-$ ponent would be identified as the dominant mode, it would be erroneously considered to be mixed with others components which arises only as result of the improper positioning of the Mirnov coils.

Summarizing, the results obtained from these series of simulations demonstrate to be consistent and clearly indicate that the introduction of the toroidal effect is quite fundamental to avoid misleading interpretations of the mode composition that one usually obtains when typical Mirnov experimental magnetic signals are Fourier analyzed.

\section{Effect Caused by the Geo- metrical Displacement of the Plasma Column}

The fluctuating poloidal magnetic field amplitude detected by the Mirnov coils is usually analyzed supposing the plasma current in tokamaks remaining properly positioned during the entire plasma discharge. However, experimental observations have already shown that this assumption is not always true. Thus, simulations were also carried out in this work with the objective of evaluating how the Fourier analysis of the measured MHD oscillations is affected when displacements of a circular plasma column occur.

Considering the magnetic pick-up coils being fixed in a radial position $r$ (within the shade of the tokamak material limiter) then it is possible to obtain a correction factor for the amplitude of the measured magnetic fields which is, in a first approximation, proportional to the horizontal/vertical plasma column displacement. This correction factor is assumed, for simplification, to be given by the ratio:

$$
f=\frac{r}{r_{1}}
$$

where $\mathrm{r}$ is the distance between the center of the original plasma column and the Mirnov coils, and $\mathrm{r}_{1}$ represents the same distance in relation to the plasma column that has been displaced, as shown in Fig. 8. Hence, if the displacement of the plasma column occurs in a given direction, towards to a particular magnetic coil, the amplitude of the detected field becomes proportionally increased according to the factor $\mathrm{f}$ given in equation 7 . 

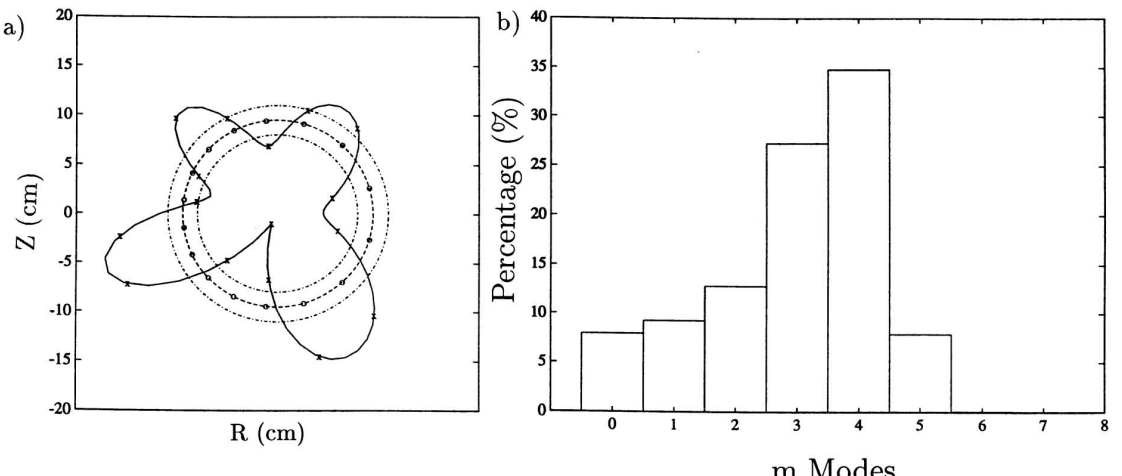

Figure 4. a) Poloidal configuration of the simulated magnetic field with dominant presences of the $\mathrm{m}=4$ and $\mathrm{m}=3$ components. The o-symbols indicate the poloidal positions of the coils with the introduction of the toroidal geometry effect and the $\mathrm{x}$-symbol indicate the signal amplitude measured by each coil. b) Mode composition, in percentage, of the simulated signal.
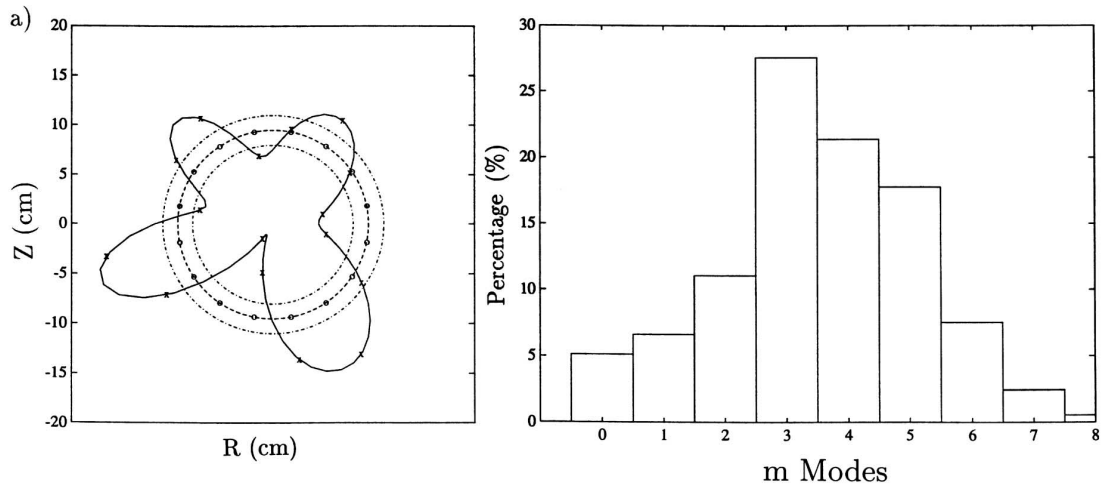

Figure 5. a) The same simulated signal of fig. 4 but with the magnetic coils positioned accordingly to the cylindrical model. Note that the coils are now equally spaced. b) Mode composition obtained for the simulated signal which shows significant difference in relation to the original signal.
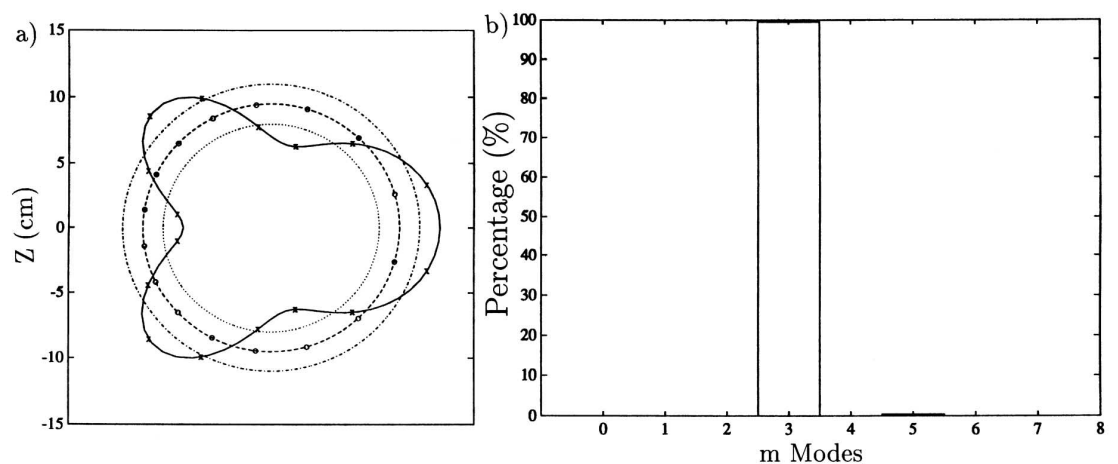

Figure 6. a) Poloidal configuration of a pure $\mathrm{m}=3$ simulated signal. b) Corresponding mode composition obtained when the toroidal geometry effect is considered 

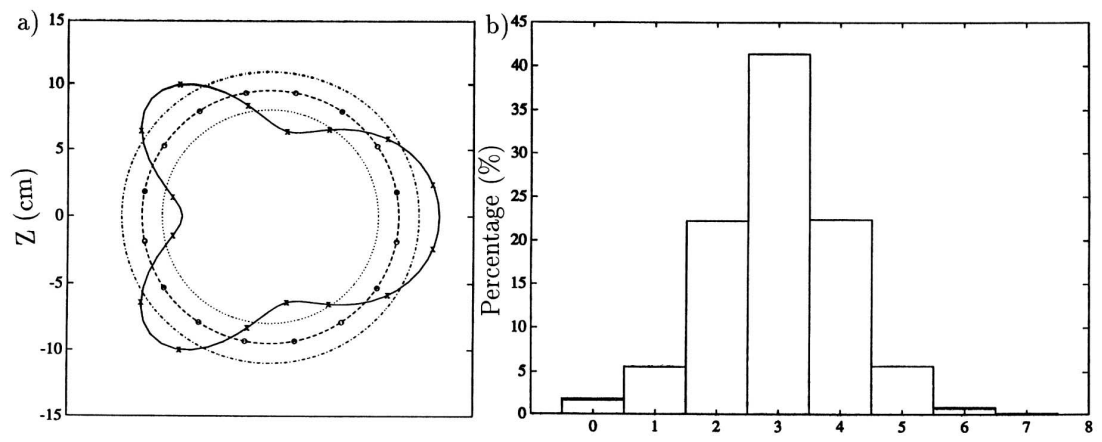

$\mathrm{m}$ Modes

Figure 7. a) The same simulated signal of Fig. 6. b) The corresponding mode decomposition in the cylindrical approximation.

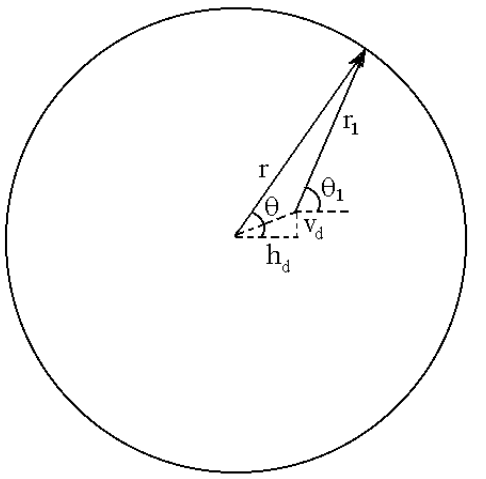

Figure 8. System of coordinates $(\mathrm{r}, \theta)$ and $\left(\mathrm{r}_{1}, \theta_{1}\right)$ used to describe the plasma column is centered, and shifted from the original position, respectively.

A convenient expression for $r_{1}$ can be easily obtained from the basic equations that relate the Cartesian to the polar system of coordinates:

$$
\begin{aligned}
& x=r \cos (\theta) \\
& y=r \sin (\theta)
\end{aligned}
$$

Therefore, in terms of the new coordinates $\left(\mathrm{r}_{1}, \theta_{1}\right)$, we have:

$$
\begin{aligned}
& x=r_{1} \cos \left(\theta_{1}\right)+h_{d} \\
& y=r_{1} \sin \left(\theta_{1}\right)+v_{d}
\end{aligned}
$$

where $\mathrm{h}_{d}$ and $\mathrm{v}_{d}$ are the horizontal and vertical components of the plasma column displacement, respectively.

The two sets of coordinates $(r, \theta)$ and $\left(\mathrm{r}_{1}, \theta_{1}\right)$ are shown in Fig. 8. The relation between them can be obtained by resolving the system of equations:

$$
r_{1}=\frac{r \sin (\theta)-v_{d}}{\sin \left(\theta_{1}\right)}
$$

where:

$$
\left.\theta_{1}=\arctan \left(\frac{r \sin (\theta)-v_{d}}{r \cos (\theta)-h_{d}}\right)\right)
$$

Therefore, after obtaining $\theta_{1}$ from equation 11 and substituting the result in equation 10 , the correction factor $\mathrm{f}$ can be properly evaluated for each displacement considered.

To investigate how possible plasma column displacements could affect the Fourier analysis of the MHD activity, a mixture of modes corresponding to the spectrum shown in Fig. 9 was chosen. Note that the $\mathrm{m}=$ 3 is the dominant component, in accordance to what is usually observed in TBR discharges [2,4]. The corresponding representation of this mode composition in polar coordinates is shown in Figs. 10a and 10b, where the full-line represents the magnetic field that would be measured with the plasma column centered, while the line-point curve corresponds to the field configuration that would be detected if the plasma column exhibits horizontal displacements. The group of small circumferences along the broken-line corresponds to the positions of 24 pick up coils related to a hypothetical Mirnov coil system.

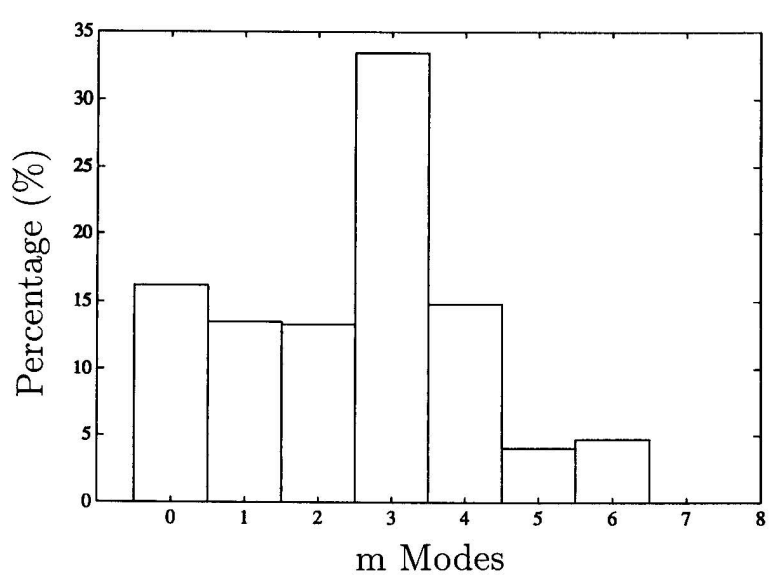

Figure 9. Mode composition of the simulated magnetic signal. 
a)

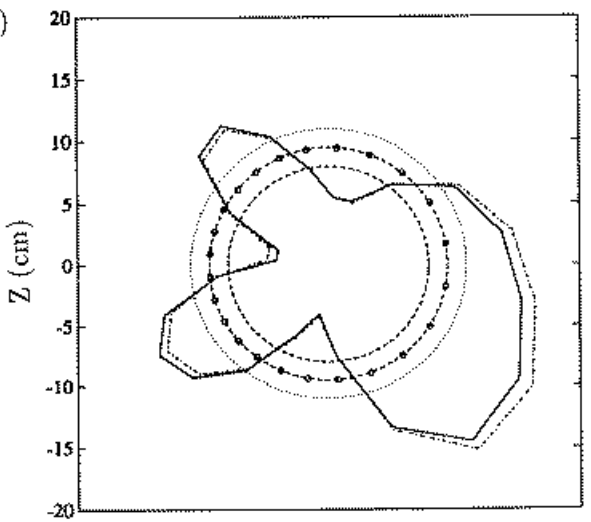

b)

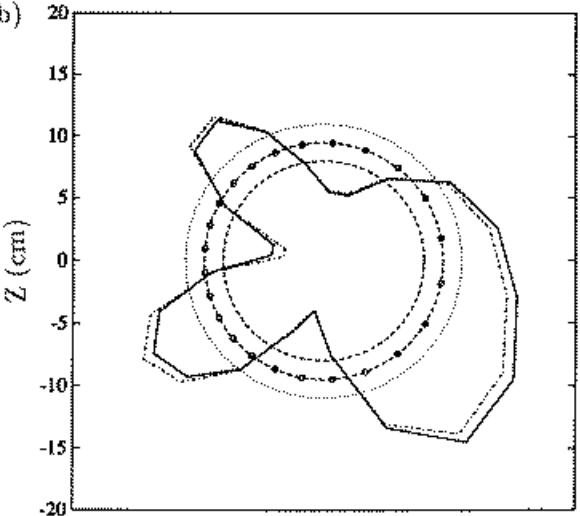

Figure 10. a) Polar representation of the poloidal magnetic field as detected by a set of 24 Mirnov coils after the plasma column has moved 1,5 cm towards the outside and b) after moving 1,5 cm towards the inside.

Since vertical displacements of the plasma column in TBR, alike other tokamaks, are commonly very small, only changes in the mode spectrum due to horizontal column displacements were analyzed. For the simulation that was carried out, the range of displacements chosen for the plasma column was $1.5 \mathrm{~cm}$ towards the outside, that is, towards the region of lower toroidal magnetic field (Fig. 10a) and $1.5 \mathrm{~cm}$ towards the inside (Fig. 10b). Thus, the magnetic field configuration for 30 different displacements (in $1 \mathrm{~mm}$ steps) was calculated: 15 displacements towards the inside $\left(\mathrm{h}_{d}<0\right)$ and 15 towards the outside $\left(\mathrm{h}_{d}>0\right)$. Afterwards, these simulated signals were Fourier analyzed and the corresponding mode spectrum, for each case, was calculated.

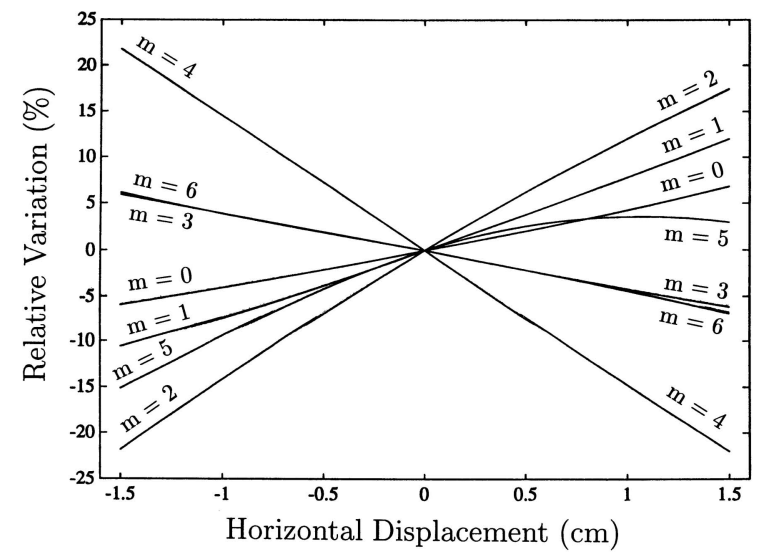

Figure 11. Relative variation of the mode composition as a function of horizontal displacements of the plasma column towards the inside (high $\mathrm{B}_{\phi}$ ) and outside (low $\mathrm{B}_{\phi}$ ) regions of the vacuum vessel.

The results obtained showed that some differences on the mode composition are clearly observed when comparing these results to the ones that would be obtained if the plasma column would be kept centered. The relative variations obtained for the amplitude of each mode is shown in Fig 11. Note from this figure that there is an almost linear dependence of the mode intensity with the plasma displacements and, what is more interesting, this variation strongly depends on the mode number.

\section{Uncertainties on the MHD Mode Intensity due to a limited number of magnetic pick-up coils}

A series of simulations was finally carried out in this work, to determine how strongly the Fourier analysis of the magnetic signals is affected by the limited number of pick up coils that is usually found in Mirnov systems. To perform this analysis, the two sets of coils that have been used in TBR-1 tokamak were considered: one with 10 coils and the other with 16 coils.

The uncertainties in the amplitude of the mode oscillations, due to limited number of detecting coils in the Mirnov systems, was investigated by simulating 30 magnetic signals, each one composed by a different combinations of modes with intensities randomly chosen. These signals were created by using the first 26 terms of the series of Fourier, represented by the equation:

$$
B\left(\theta^{*}\right)=\sum a_{m} \cos \left(m \theta^{*}\right)+b_{m} \sin \left(m \theta^{*}\right)
$$

where $\mathrm{a}_{m}$ and $\mathrm{b}_{m}$ are the Fourier coefficients corresponding to the poloidal components, and $\theta^{*}$ it is the parameter that was defined previously in equation 3 . Therefore, 14 different modes ranging from $\mathrm{m}=0$ to $\mathrm{m}$ $=13$ should be discriminated by the coil systems.

The mode amplitudes obtained from the Fourier analysis of the simulated signals were compared to the 
original ones and the average of the relative errors, in percentage, are shown in Table 1, for both coil systems. Note from this table that the uncertainties are somewhat larger for modes with larger $\mathrm{m}$ and, as expected, the system composed of 16 coils yields more accurate results than the system with only 10 coils. This accuracy is especially important for investigating the occurrence of MHD instabilities in tokamak plasmas since the lower mode numbers perturbations are the ones usually held responsible for triggering these instabilities.

Table 1

\begin{tabular}{|c|c|c|}
\hline $\begin{array}{c}\text { Mode } \\
\text { Number }\end{array}$ & $\begin{array}{c}\text { System 1 } \\
\text { 10 coils (\%) }\end{array}$ & $\begin{array}{c}\text { System 2 } \\
\mathbf{1 6} \text { coils (\%) }\end{array}$ \\
\hline $\mathrm{m}=0$ & 16.1 & 0.0 \\
\hline $\mathrm{m}=1$ & 12.1 & 0.0 \\
\hline $\mathrm{m}=2$ & 9.0 & 0.0 \\
\hline $\mathrm{m}=3$ & 10.0 & 0.6 \\
\hline $\mathrm{m}=4$ & 27.3 & 4.5 \\
\hline $\mathrm{m}=5$ & 43.3 & 9.0 \\
\hline $\mathrm{m}=6$ & - & 12.5 \\
\hline $\mathrm{m}=7$ & - & 22.4 \\
\hline $\mathrm{m}=8$ & - & 35.9 \\
\hline
\end{tabular}

\section{Conclusions}

The series of simulations carried out in this work have shown that toroidal geometry effects cannot be neglected when the experimental poloidal magnetic field fluctuations in tokamaks are Fourier analyzed. Basically, it was observed the existence of a strong phase modulation on the perturbed magnetic signals, which amplitude is directly proportional to the inverse of the tokamak aspect ratio $(\varepsilon)$. Since the intensity of the fluctuating magnetic field was observed to vary more rapidly within the higher toroidal magnetic field side of the tokamak, then it can be concluded that a larger number of magnetic pick-up coils must be installed around this region, in order to improve the detection spatial resolution.

Comparing the results obtained by Fourier analyzing the simulated magnetic signals, with and without considering the effects caused by the toroidal geometry, it was observed that the significant differences found between the two cases indicate that toroidal geometry effects have to be taken into account for the correct determination of the mode composition.

Also, an evaluation of how the Fourier analysis would be affected by the occurrence of possible horizontal displacements of the plasma column was performed. Although it was observed that plasma column shifts can lead to an incorrect mode decomposition of the fluctuating poloidal magnetic signals this effect, if ignored, does not affect the results as strongly as when the toroidal geometry effect is disregarded.

Finally, it was shown that the accuracy of the results obtained would depend significantly on the number of Mirnov coils used to measure the oscillating poloidal magnetic field. It was observed that even for lower $\mathrm{m}$ modes a considerable number of coils are necessary to be used if the mode composition is to be accurately obtained.

\section{References}

[1] S.V. Mirnov, Nucl. Fusion 9, 57 (1969).

[2] A. Vannucci, I.C. Nascimento, I.L. Caldas, Plasma Phys. Contr. Fusion 31, 147 (1989).

[3] Z.A. Pietrzyk et. al., Nucl. Fusion 32, 1735 (1992).

[4] M.S.T. Araujo, A. Vannucci, I.L. Caldas, Il Nuovo Cimento 18D, 807 (1996).

[5] A. Vannucci, S.C. McCool, Nucl. Fusion 37, 1229 (1997).

[6] S. Turlur, E. Joffrin, M. Zabiego, Ph. Ghendrih, A. Grosman, Jour. Nucl. Materials 241-243, 548 (1997).

[7] S. Tsuji et al., Nucl. Fusion 25, 305 (1985).

[8] A. Vannucci, R.D. Gill, Nucl. Fusion 31, 1127 (1991).

[9] G. Pautasso et. al., Nucl. Fusion 36, 1291 (1996).

[10] H.R. Koslowski et al., Nucl. Fusion 40, 821 (2000).

[11] T.R. Harley, D.A. Buchenauer, J.W. Coonrod, K.M.McGuire, Nucl. Fusion 29, 771 (1989).

[12] G. Hammett, K.M. McGuire, Princeton Plasma Physics Laboratory Report PPPL-1854, 1982 (unpublished).

[13] V.G. Merezhkin, Sov. Jour. Plasma Phys. 4, 152 (1978).

[14] A. Boozer, A.B. Rechester, Phys. Fluids 21, 682 (1978). 